\title{
On the homeostatizing of perturbations (from pupillary dynamics)
}

\section{Wouter E. Boekel}

Pupillary dynamics have been established to inform us about a range of behaviours such as mind-wandering (Smallwood et al., 2011, Mittner et al., 2014, 2016) and trust (Kret et al., 2015, Prochazkova et al., 2018). One of the most general hypotheses of the underlying mechanisms is that pupil diameter changes signal fluctuations in arousal by way of fluctuations in norepinephrine signaling from the locus coeruleus (Aston-Jones and Cohen, 2005). In neural network dynamics, the default mode network in interaction with locus-coeruleus norepinephrine (LC-NE) forms a homeostatizing role for many other intrinsic connectivity networks (Mesulam, 2008, Braga and Leech, 2015, Eldar et al., 2013). Neural annealing through arousal fluctuations by way of LC-NE signalling may allow the network to ever more quicker reach homeostasis in a stable state of high complexity. Generally the brain appears to aim for the homeostatizing of perturbations arriving on its sensory manifold. While the brain is usually hypothesized to reconstruct an internal representation of the three-dimensional (4D when taking into account the representation of more than a single 3D state, i.e. more than one moment, i.e. trajectories in memory) external world by way of a 3D delta matrix, the sensory manifold is more accurately modeled by a 2D torus. Trajectory perturbations on this torus may then be viewed as having four dimensions (two spatial coordinates, inward/outward perturbations, and multiple sequential occurrences). Part of the initial dimensional confusion may arise from this difference between our inference of the external 3D world, and our 2D sensory manifold. As the brain is hypothesized generally to homeostatize perturbations, handling a 2D toroidal model allows a more direct and therefore quicker homeostatizing of perturbations than handling a 3D rectilinear model. The brain's measuring of these trajectories of perturbations allows it to anticipate future occurrences of similar trajectories of perturbations, opening the possibility of sending counter-perturbations so as to preserve the non-perturbed state of the toroidal model. That is how the brain homeostatizes perturbations on a toroidal model of the sensory manifold. When a perturbation trajectory is homeostatized only partly by way of the re-occurrence of the perturbation not entirely overlapping with the initially measured perturbation, two things happen: the remaining perturbation is again measured, and an attempt is made at further homeostatizing. Secondly, the perturbation prior to the initial attempt at homeostatizing is reconstructed and added to memory so as to allow keeping track of commonly occurring perturbation trajectories. Over longer timescales this leads to the homeostatizing of distributions of perturbation trajectories rather than single perturbation trajectories. Similarly to the first procedure of homeostatizing a leftover perturbation, residual distributions of perturbation trajectories are iteratively homeostatized. This relates to predictive coding (Huang et al., 2011), bayesian brain (Knill and Pouget, 2014), free energy principle (Friston, 2010) and projective consciousness (Rudrauf et al., 2017). It shows how the brain performs statistical analyses of re-occurring perturbations of the sensory manifold so as to sublimate future occurrences of perturbations into toroidal homeostasis, offering the experience of pure sensory awareness. The accumulation of pure sensory awareness leads to its clear-minded consciousness. This then leads to the observing of the process of homeostatizing of toroidal perturbations, finally allowing its description, its description in turn allowing its own homeostatizing, iteratively into clear sensory awareness.

\section{Literature}

Smallwood, J., Brown K. S., Tipper, C., Giesbrecht, B., Franklin, M. S., Mrazek, M. D., Carlson, J. M., Schooler, J. W. (2011) Pupillometric Evidence for the Decoupling of Attention from Perceptual Input during Offline Thought. PLoS ONE, 6(3). 
Mittner, M., Boekel, W., Tucker, A. M., Turner, B. M., Heathcote, A., Forstmann, B. U. (2014) When the Brain Takes a Break: A Model-Based Analysis of Mind Wandering. The journal of Neuroscience, 34(49):16286-16295.

Mittner, M., Hawkins, G. E., Boekel, W., Forstmann, B. U. (2016) A Neural Model of Mind Wandering. Trends in Cognitive Sciences, 20(8):570-578.

Kret, M. E., Fischer, A. H., de Dreu., C. K. W. (2015) Pupil Mimicry Correlates With Trust in InGroup Partners With Dilating Pupils. 26(9):1401-1410.

Prochazkova, E., Prochazkova, L., Giffin, M. R., Scholte, H. S., de Dreu, C. K. W., Kret, M. E. (2018) Pupil mimicry promotes trust through the theory-of-mind network. 115(31):E7265E7274.

Aston-Jones, G., Cohen, J. D. (2005) An Integrative Theory of Locus Coeruleus-Norepinephrine Function: Adaptive Gain and Optimal Performance. Annu Rev. Neurosci. 28:403-50.

Mesulam, M. M. (1988) From sensation to cognition. Brain, 121:1013-1052.

Mesulam, M. M. (2008) Representation, Inference, and Transcendent Encoding in Neurocognitive Networks of the Human Brain. Ann Neurol, 64:367-378.

Braga, R. M., Leech, R. (2015) Echoes of the Brain: Local-Scale Representation of Whole-Brain Functional Networks within Transmodal Cortex. The Neuroscientist. 21(5):540-551.

Eldar, E., Cohen, J. D., Niv, Y. (2013) The effects of neural gain on attention and learning. Nature Neuroscience. 16(8):1146-1156.

Huang, Y., Rao, R. P. N. (2011) Predictive coding. Wiley Interdisciplinary Reviews: Cognitive Science 2(5):580-593.

Knill, D. C., Pouget, A. (2004) The Bayesian brain: the role of uncertainty in neural coding andcomputation. TRENDS in Neuroscience. 27(12):712-719

Friston, K. (2010) The free-energy principle: a unified brain theory? Nature reviews neuroscience. (11):127-138.

Rudrauf, D., Bennequin, D., Granic, I., Landini, G., Friston, K., Williford, K. (2017) A mathematical model of embodied consciousness. Journal of Theoretical Biology. 428:106-131. 